\title{
An overview of CALET observations after three years on the International Space Station
}

\author{
Pier Simone Marrocchesi for the CALET Collaboration * \\ Dept.of Physical Sciences, Earth and Environment, Univ. of Siena, 53100 Siena, Italy \\ E-mail: marrocchesiepi.infn. it, for the CALET Collaboration ${ }^{\dagger}$
}

\begin{abstract}
The CALorimetric Electron Telescope CALET is a space-based instrument designed to carry out precision measurements of high energy cosmic-rays on the JEM-EF external platform of the ISS where it has been collecting science data continuously since mid October 2015. Equipped with a thick $\left(30 \mathrm{X}_{0}\right.$ and $\sim 1.3 \lambda_{I}$ ) calorimeter with an imaging pre-shower and with two independent subsystems to identify the charge of the incident particle, CALET has the depth, tracking capability, electron/proton discrimination and energy resolution to study hadrons, electrons and gamma rays in the cosmic radiation. An overview of CALET observations is presented, based on the data taken during the first three years. It includes a direct measurement of the electron+positron energy spectrum from $11 \mathrm{GeV}$ to $4.8 \mathrm{TeV}$ in good agreement with AMS-02 data in the region below $\sim 1 \mathrm{TeV}$ and suggesting a flux suppression above $1 \mathrm{TeV}$. In the energy region below $\sim$ $300 \mathrm{GeV}$, CALET's spectral index is consistent with AMS-02, Fermi-LAT and DAMPE, while from $\sim 300 \mathrm{GeV}$ to $600 \mathrm{GeV}$ the spectrum is significantly softer than the spectra from the latter two experiments. The proton spectrum has been measured from $50 \mathrm{GeV}$ to $10 \mathrm{TeV}$ covering, for the first time with a single space-borne instrument, the whole energy interval previously investigated in separate sub-ranges by magnetic spectrometers and calorimetric instruments. The observed spectrum is consistent with AMS-02 but it extends by nearly one order of magnitude higher in energy, showing a smooth transition of the power-law spectral index from $-2.81 \pm 0.03$ $(50-500 \mathrm{GeV})$ to $-2.56 \pm 0.04(1-10 \mathrm{TeV})$, thereby providing evidence of a deviation from a single power law by more than 3 sigma. In addition to its primary goal of identifying nearby sources of high-energy electrons and possible signatures of dark matter in the electron spectrum, CALET is carrying out extensive measurements of the energy spectra, relative abundances and secondaryto-primary ratios of elements from proton to iron and above (up to $\mathrm{Z}=40$ ) studying the details of galactic particle propagation and acceleration. Preliminary spectra of cosmic-ray nuclei are presented, together with gamma-ray observations and searches of an e.m. counterpart of LIGO/Virgo GW events.
\end{abstract}

European Physical Society Conference on High Energy Physics - EPS-HEP2019 10-17 July, 2019

Ghent, Belgium

\footnotetext{
*Speaker.

${ }^{\dagger}$ for collaboration list see PoS(ICRC2019) 1177
} 


\section{Introduction}

CALET is a space mission led by the Japanese Space Agency (JAXA) with the participation of the Italian Space Agency (ASI) and NASA. It was launched on August 19, 2015 with the Japanese carrier H-II, delivered to the ISS by the HTV-5 Transfer Vehicle, and installed (Fig.1(a)) on the Japanese Experiment Module Exposure Facility (JEM-EF). The CALET science program addresses several outstanding questions of high-energy astroparticle physics including the origin of cosmic rays (CR); the possible presence of nearby astrophysical CR sources; the study of their acceleration mechanism(s); the propagation of primary and secondary elements in the galaxy; the nature of dark matter and its localization. The design of the CALET instrument $[1,2]$ has been optimized for high precision measurements of the electron spectrum allowing an accurate scan of the energy interval already covered by previous experiments [3] and its extension to the unexplored region above $1 \mathrm{TeV}$. Given the high energy resolution of CALET, a detailed study of the spectral shape might reveal the presence of nearby sources of acceleration as well as possible indirect signatures of dark matter. Taking advantage of its capability of identifying cosmic rays with in-

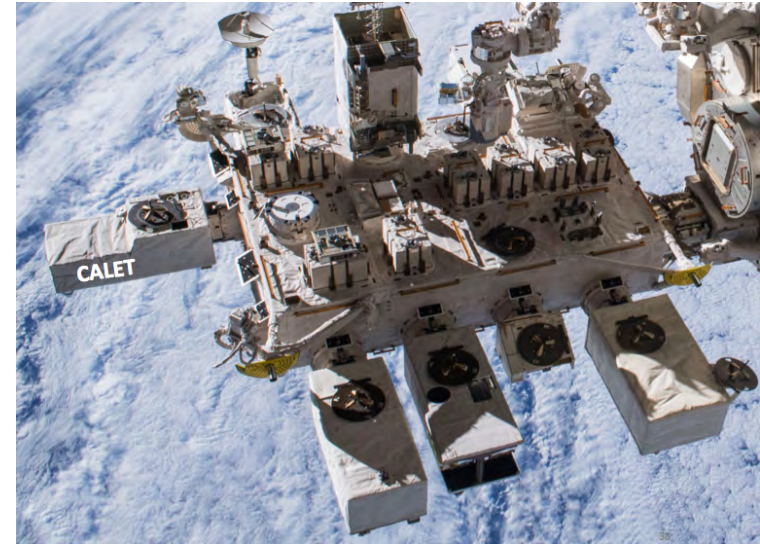

(a)
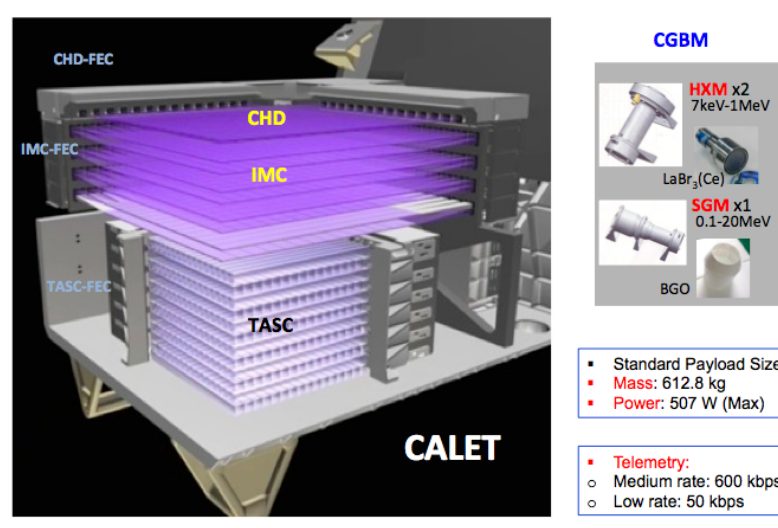

(b)

Figure 1: (a) CALET on the JEM-EF; (b) Layout of the CALET instrument and of the Gamma-Ray Burst Monitor (CGBM)

dividual element resolution, CALET is also carrying out direct measurements of the spectra and relative abundances of light and heavy cosmic nuclei $[28,29]$ from proton to iron in the energy range from a few tens of $\mathrm{GeV}$ to several hundred TeV. The abundances (normalized to Iron) of trans-iron elements up to $\mathrm{Z} \sim 40$ are studied with a dedicated program of long term observations [30]. CALET can identify gamma-rays and measure their energies from $\sim 1 \mathrm{GeV}$ to the $\mathrm{TeV}$ region with an angular resolution $<2^{\circ}$ above $1 \mathrm{GeV}\left(<0.2^{\circ}\right.$ above $\left.10 \mathrm{GeV}\right)$ as summarized in the last chapter.

\section{CALET (CALorimetric Electron Telescope)}

The CALET instrument (Fig 1(b)) includes a thick calorimeter, designed to achieve shower containment and a large proton rejection capability $\left(>10^{5}\right)$, which is longitudinally segmented into a fine grained imaging calorimeter (IMC) followed by a total absorption calorimeter (TASC). The TASC is a $27 \mathrm{X}_{0}$ thick homogeneous calorimeter with 12 alternate $\mathrm{X}-\mathrm{Y}$ layers of lead-tungstate (PWO) logs. The IMC is a sampling calorimeter segmented longitudinally into 16 layers of scintillating fibers (with $1 \mathrm{~mm}^{2}$ square cross-section) readout individually and interspaced with thin tungsten absorbers. Alternate planes of fibers are arranged along orthogonal directions. It can image the early shower profile in the first $3 \mathrm{X}_{0}$ and reconstruct the incident direction of cosmic rays with good angular resolution $\left(0.1^{\circ}\right.$ for electrons and better than $0.5^{\circ}$ for hadrons) $[1,3]$. The overall thickness of CALET at normal incidence is $30 \mathrm{X}_{0}$ and $\sim 1.3$ proton interaction length $\left(\lambda_{I}\right)$. 


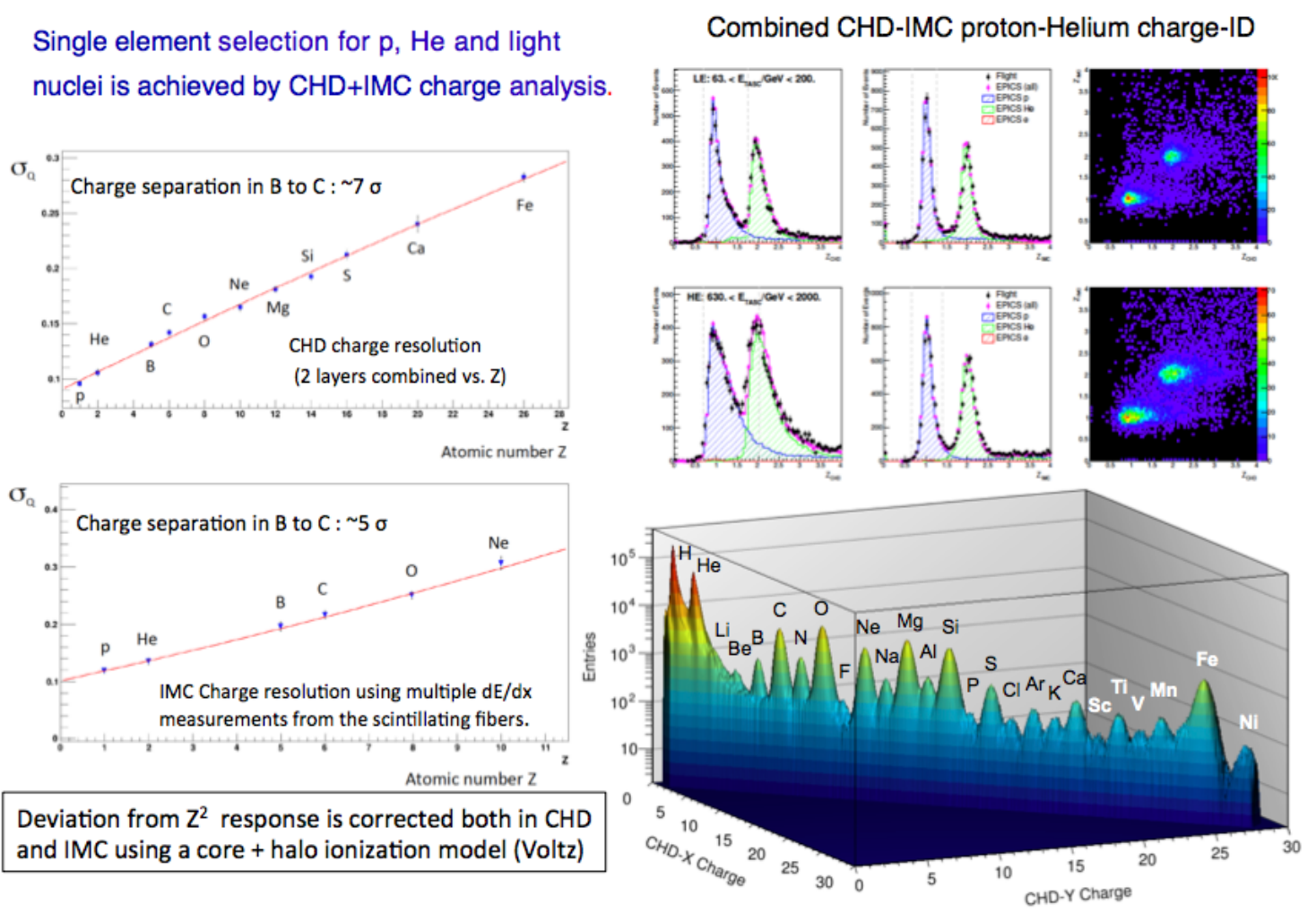

Figure 2: Top left panel: CHD charge resolution for elements from proton to iron; Bottom left panel: IMC charge resolution (proton to neon); Correlation plots of CHD vs IMC charge are shown: (i) for proton and helium candidates (top right panels) (ii) for elements from proton to nichel (bottom right panel)

The charge identification of individual nuclear species is performed by a two-layered hodoscope of plastic scintillators (CHD), positioned at the top of the apparatus, providing a measurement of the charge $Z$ of the incident particle over a wide dynamic range $(Z=1$ to $\sim 40)$ with sufficient charge resolution to resolve individual elements [33] and complemented by a redundant charge determination via multiple $\mathrm{dE} / \mathrm{dx}$ measurements in the IMC. The overall $\mathrm{CHD}$ charge resolution (in $\mathrm{Z}$ units) is plotted in Fig.2(a) showing a linear increase as a function of the atomic number from $<0.1$ for protons to $\sim 0.3$ for iron. For the IMC, although the photostatistics available at single fiber level is about one order of magnitude lower than a single CHD layer, the charge measurement - thanks to the multiple sampling - achieves an adequate performance as shown in Fig.2(b) where the IMC charge resolution is plotted as a function of atomic number $\mathrm{Z}$. The interaction point (IP) is first reconstructed [37] and only the $\mathrm{dE} / \mathrm{dx}$ ionization clusters from the layers upstream the IP are used. The charge value is evaluated as the truncated-mean of the valid samples. The geometrical factor of CALET is $\sim 0.1 \mathrm{~m}^{2} \mathrm{sr}$ and the total weight is $613 \mathrm{~kg}$. The instrument is described in more detail elsewhere [1,34].

\section{Direct Measurements on the ISS with CALET}

\subsection{Inclusive Electron Spectrum}

The CALET collaboration reported their first measurement of the inclusive electron+positron spectrum in the energy range from $10 \mathrm{GeV}$ to $3 \mathrm{TeV}$ [25]. Soon after, the DArk Matter Particle Explorer (DAMPE) collaboration published their all-electron spectrum in the energy interval from $25 \mathrm{GeV}$ to $4.6 \mathrm{TeV}$ [26]. The latter publication was followed by many papers speculating about the 
origin of a peak-like structure near $1.4 \mathrm{TeV}$ in the DAMPE data. An updated version of the CALET all-electron spectrum (Figure 3) using 780 days of flight data and the full geometrical acceptance was published covering the energy range from $11 \mathrm{GeV}$ to $4.8 \mathrm{TeV}$ [27].

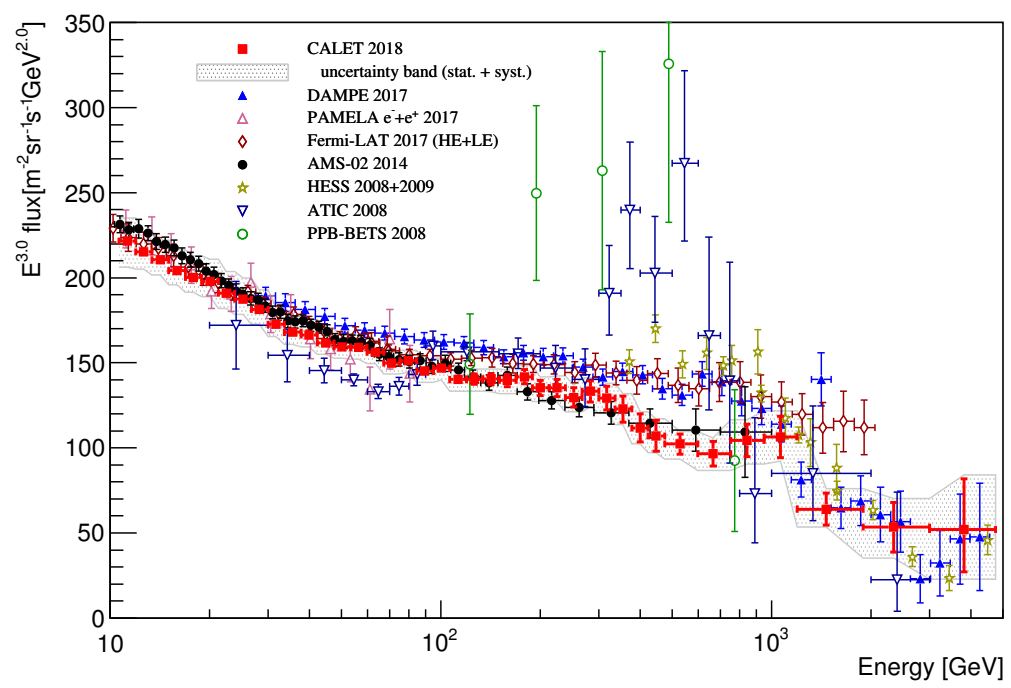

Figure 3: Cosmic-ray all-electron spectrum measured by CALET from $10.6 \mathrm{GeV}$ to $4.75 \mathrm{TeV}$ [27]. The gray band indicates the quadratic sum of statistical and systematic errors (not including the uncertainty on the energy scale). Also plotted are direct measurements in space including [26, 16, 6, 10, 7, 8] and from ground-based experiments [9].

The width of each bin is shown as a horizontal bar, the statistical errors as vertical bars, while the gray band is representative of the quadratic sum of statistic and systematic errors. A comprehensive study of the systematic uncertainties was performed as described in Refs. [25, 27] and Supplemental Material therein. Taking the currently available experimental data at face-value we notice that the all-electron spectrum data seem to fork into two groups of measurements: AMS-02 + CALET and Fermi/LAT + DAMPE, with good consistency within each group, but with only marginal overlap between the two, possibly indicating the presence of unknown systematic errors. CALET spectrum is consistent with AMS-02 below $\sim 1 \mathrm{TeV}$ where both experiments have a good electron identification capability albeit using different detection techniques. CALET observes a flux suppression above $\sim 1 \mathrm{TeV}$ consistent with DAMPE within errors. No peak-like structure was found at $1.4 \mathrm{TeV}$ in CALET data, irrespective of energy binning. After rebinning with the same set of energy bins as DAMPE, an inconsistency between the two measurements emerges with a $4 \sigma$ significance. The latter includes the systematic errors quoted from both experiments. Possible binning related effects in the CALET all-electron spectrum were also investigated by introducing a shift by $1 / 4$ of the bin width. The deviation ascribed to the binning is well below CALET energy dependent systematic uncertainty or statistical fluctuations. Therefore, bin-to-bin migration and related effects turn out to be negligible compared with the estimated systematic uncertainties, as expected from the estimated CALET energy resolution of $2 \%$ above $20 \mathrm{GeV}$. The solid curves in Figure 3 show the energy dependent systematic uncertainty band.

\subsection{Proton Spectrum}

Proton energies from $\mathrm{GeV}$ to the multi-TeV region (Fig.4) have been explored - in most cases in separate subranges - by both magnetic spectrometers (BESS-TeV, PAMELA, and AMS-02) and calorimetric instruments (ATIC, CREAM, and NUCLEON). In the intermediate energy region from 
$200 \mathrm{GeV}$ to $800 \mathrm{GeV}$ a deviation from a single power-law has been observed for both proton and helium spectra by CREAM [19, 21, 22], PAMELA [23, 24] and confirmed with high statistics measurements by AMS-02 [17]. The recent observations by AMS-02, in the region below maximum detectable rigidity (MDR) of a few TV, clearly show that primary elements (like helium, carbon and oxygen) have a very similar rigidity dependence above $\sim 60 \mathrm{GV}$ and that secondary elements (like $\mathrm{Li}, \mathrm{Be}$ and $\mathrm{B}$ ) also share an almost identical dependence, albeit different from the primaries. While the latter behavior could probably be attributed to propagation effects (secondaries propagate first as primaries and then as secondaries) it is very important to investigate with high accuracy the energy dependence of the spectral index by extending the presently available measurements to the multi- $\mathrm{TeV}$ region.

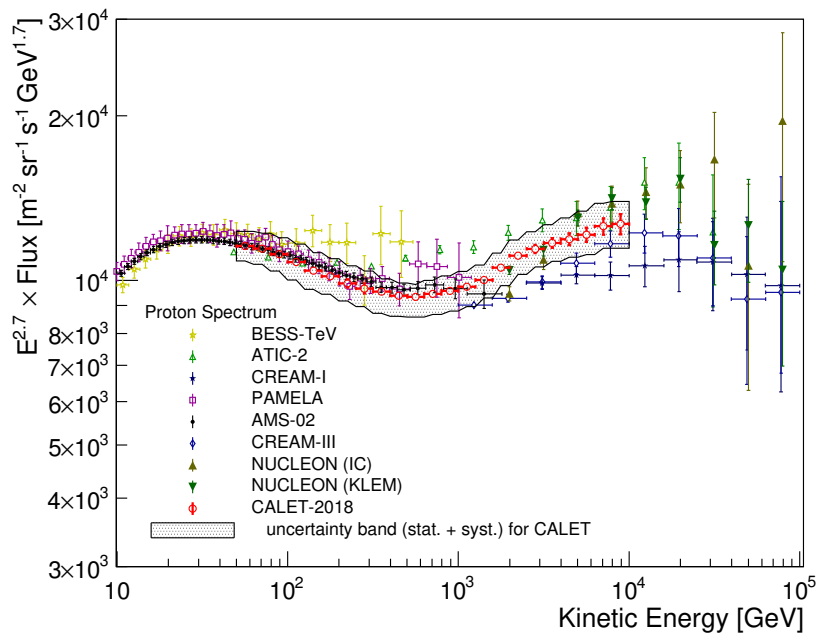

(a)

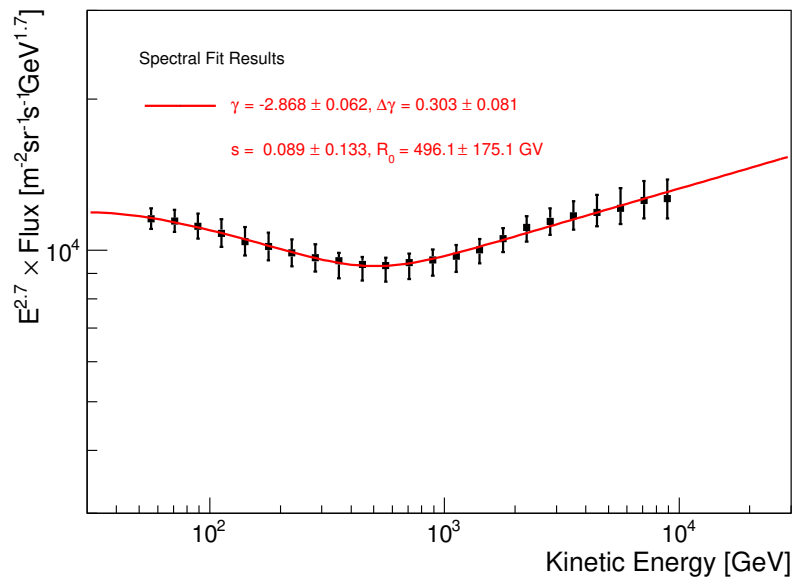

(b)

Figure 4: (a) Cosmic-ray proton spectrum measured by CALET from $50 \mathrm{GeV}$ to $10 \mathrm{TeV}$ using an energy binning of 10 bins per decade. The gray band indicates the quadratic sum of statistical and systematic errors. Also plotted are recent direct measurements in space and from high altitude balloons $[17,11,22,19,12,13,14,15]$ (b) Fit of the CALET proton spectrum with a smoothly broken power-law function as defined in Eq. (3) of Ref. [17]. Statistical errors are shown in quadrature with systematic errors including only energy dependent ones, neglecting to first order possible correlations among different sources of systematic errors and assuming that they are normally distributed. The fit parameters are given in the plot.

Taking advantage of its large dynamic range, CALET has been able to cover the whole interval of proton energies from $50 \mathrm{GeV}$ to $10 \mathrm{TeV}$ with a single space-borne instrument. This is of particular relevance because CR flux measurements are well known to be affected by relatively large systematic errors often specific of each instrument. The proton flux was extracted from the data collected from October 13, 2015 to August 31, 2018 (1054 days) on the ISS using only 40\% of the total acceptance. CALET observations (Fig.4)(a) are consistent with AMS-02 but extend to nearly one order of magnitude higher in energy, showing a very smooth transition of the power-law spectral index from $-2.81 \pm 0.03(50-500 \mathrm{GeV})$ neglecting solar modulation effects (or $-2.87 \pm 0.06$ when including solar modulation effects in the lower energy region) to $-2.56 \pm 0.04(1-10 \mathrm{TeV})$, thereby confirming the existence of a spectral hardening and providing evidence of a deviation from a single power law by more than $3 \sigma$.

The acceleration limit of supernova remnants calculated with nominal parameters is typically found to far smaller than the energy of the "knee" and possible Z-dependent (or A-dependent) cutoffs in the spectra of nuclei are hypothesized. According to some models (e.g. [38, 39]) a flux reduction for protons and helium might be expected at rigidities as low as tens of TV. Therefore, precise direct observation of proton and helium spectra in the multi- $\mathrm{TeV}$ energy region are of extreme interest. However, exploring this uncharted territory requires a good control of the systematc 
errors, a requirement that challenges our present knowledge of hadronic interactions, backscattering and energy scale assessment at these very high energies.

\subsection{Cosmic-Ray Nuclei Spectra}

CALET is carrying out extensive measurements of the energy spectra, relative abundances and secondary-to-primary ratios of elements from proton to iron and above. CALET is investigating the intermediate energy region from $200 \mathrm{GeV} / \mathrm{A}$ to $800 \mathrm{GeV} / \mathrm{A}$ where a deviation from a single powerlaw has been observed for both proton and helium spectra by CREAM [21],[22],[19], PAMELA [23, 24] and confirmed with high statistics measurements by AMS-02 [17] that reported a similar behavior also for Li and other light nuclei. CALET is performing an accurate scan of this energy region to verify the hypothesis of a progressive hardening of the proton spectrum by measuring accuratetely the dependence of the spectral index as a function of energy. By correlating the charge measurements from the two layers of CHD (Fig.2) and the independent charge measurement by IMC, well separated charge peaks emerge on top of a low background for individual elements.

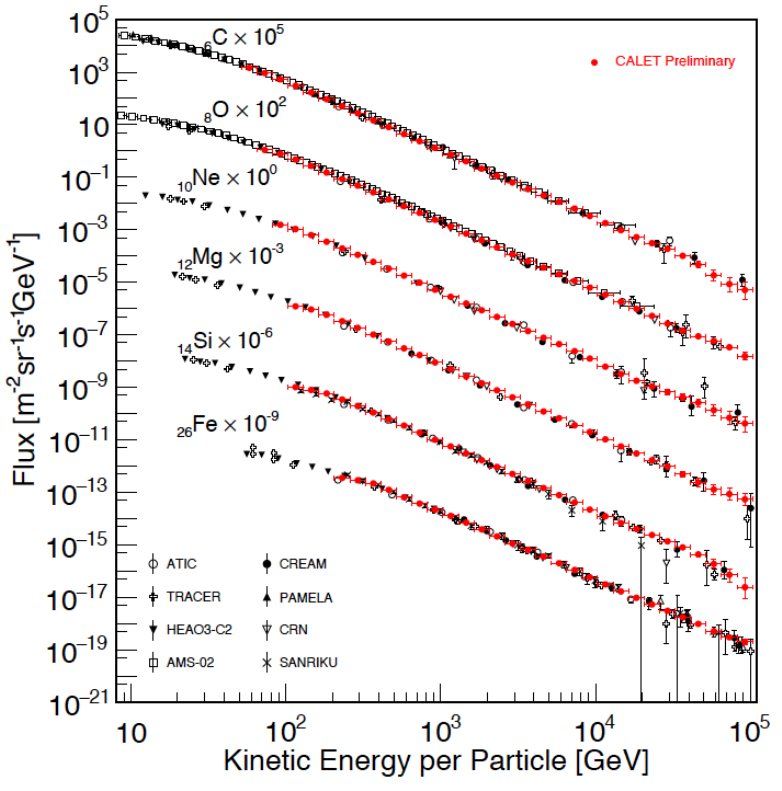

(a)

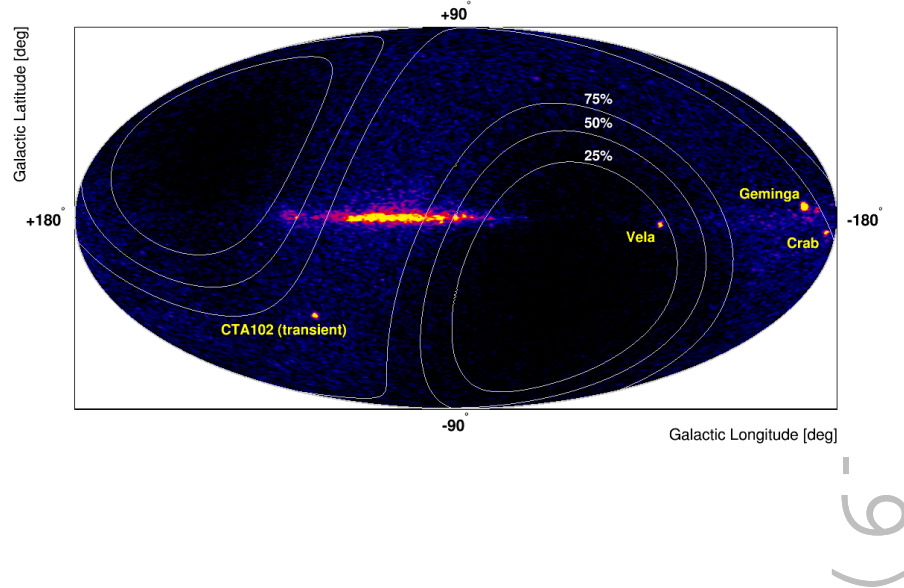

(b)

Figure 5: (a) Preliminary energy spectra of carbon, oxygen, neon, magnesium, silicon and iron as a function of kinetic energy per particle compared with previous observations. Only statistical errors are shown. (b) Gamma-ray sky map shown in a Mollweide projection of galactic coordinates. White contours show the relative level of exposure compared to the maximum on the sky.

Taking advantage of the excellent charge identification capability and wide charge span, preliminary results from the current analysis of nuclei have been presented $[40,41]$ including the spectra of carbon, oxygen, neon, magnesium, silicon and iron as shown in Fig.5(a) as a function of kinetic energy per particle.

\subsection{Observation of Gamma-Rays}

CALET can identify gamma-rays and measure their energies up to the $\mathrm{TeV}$ region. Both $\mathrm{CHD}$ and the first IMC layers are used as anti-coincidence against incoming charged particles, taking advantage of the high granularity of the IMC. In addition to the HE trigger, CALET uses a LE- $\gamma$ trigger extending the sensitivity to gamma rays with primary energies down to $\sim 1 \mathrm{GeV}$. The first 24 months data allowed a complete characterization of the performance of the instrument [44], the determination of the effective area, Point Spread Function (PSF) and absolute pointing accuracy. 
The gamma-ray sky observed by CALET using the LE- $\gamma$ trigger is shown in Fig. 5(b). Measured signals from gamma-ray bright point sources and diffuse galactic emission were found to be in agreement with simulated results and expectations from Fermi-LAT data [44]. Source spectra for Crab, Geminga, and Vela pulsars measured by CALET were tested for consistency with parameterized LAT spectra. CALET can also detect gamma-ray transients by means of the dedicated Calet Gamma-Ray Burst Monitor (CGBM) in the energy range of $7 \mathrm{keV}-20 \mathrm{MeV}$. As of June 2019, 159 GRBs have been detected, $12 \%$ of which were classified as short, with an average rate of $\sim 43$ /year. Combined analyses of the CGBM and calorimeter were performed to search for electromagnetic counterparts of gravitational waves $(\mathrm{GW})$ triggered by LIGO/Virgo. Possible signals compatible with gamma-ray emission were searched for in the calorimeter and CGBM data in time intervals of tens of seconds centered on the reported trigger times of GW151226, GW170104, GW170608, GW170814, and GW170817 events. No signal was detected for all GW events. Upper limits on gamma-ray emission were set for GW151226 (CAL + CGBM) and GW170104 (CAL), while GW170608, GW170814, GW170817 were found to be outside the CALET field-of-view [42, 43].

\section{References}

[1] S. Torii et al., Proceedings of Science (ICRC2017) 1092, 2017

[2] Y. Asaoka for the CALET collaboration, Proc. of the 36th ICRC (Madison), 2019

[3] S. Torii for the CALET collaboration, Proc. of the 36th ICRC (Madison), 2019

[4] O. Adriani et al. (CALET Collaboration), Phys. Rev. Lett. 119, 181101, 2017

[5] O. Adriani et al. (CALET Collaboration), Phys. Rev. Lett. 120, 261102, 2018

[6] Abdollahi, S.,et al. (The Fermi-LAT Collaboration) 2017, Phys. Rev. D 95082007 (16pp)

[7] Chang, J.et al. (ATIC Collaboration) 2008, Nature 456362 (4pp)

[8] Yoshida, K., et al. (PPB-BETS Collaboration) 2008, Advances in Space Research 42 1670-1675

[9] Aharonian F., et al.(H.E.S.S. Collaboration) 2009, Astron Astrophys. 508561 (4pp)

[10] Aguilar, M.,et al. (AMS Collaboration) 2014, Phys. Rev. Lett. 113221102 (9pp)

[11] A. Panov et al., Bull. Russ. Acad. Sci. Phys. 71, 494, 2007

[12] E. Atkin et al., JETP Letters 108, 5, 2018

[13] S. Haino et al., Phys. Lett. B 594, 35, 2004

[14] O. Adriani et al., Phys. Rept. 544, 323, 2014

[15] O. Adriani et al., Riv. Nuovo Cim. 40, 1, 2017

[16] Adriani, O.,et al. (PAMELA Collaboration) 2017, La Rivista del Nuovo Cimento 40473 (53pp)

[17] M. Aguilar et al., Phys. Rev. Lett. 114, 171103, 2015

[18] M. Aguilar et al., Phys. Rev. Lett. 115, 211101, 2015

[19] Y. S. Yoon et al., Astrophys. J. 839, 5, 2017

[20] O. Adriani et al. (CALET Collaboration), Phys. Rev. Lett. 122, 181102, 2019

[21] H. S. Ahn et al., Astrophys. J. 714, 89-93, 2010

[22] Y. S. Yoon et al., Astrophys. J. 728, 122, 2011

[23] O. Adriani, et al., Science 332, 69, 2011

[24] O. Adriani, et al., Astrophys. J. 765, 91-98, 2013

[25] Adriani, O., et al. (CALET Collaboration) 2017, Phys. Rev. Lett. 119181101 (6pp)

[26] Ambrosi G., et al. (DAMPE Collaboration) 2017, Nature 552 63-66

[27] Adriani, O., Akaike, et al. (CALET Collaboration) 2018, Phys. Rev. Lett. 120261102 (7pp)

[28] P. S. Marrocchesi, arXiv:1704.00304 [astro-ph.HE], 2017

[29] Y. Akaike for the CALET collaboration, Proc. of the 36th ICRC (Madison), 2019

[30] B. F. Rauch for the CALET collaboration, Proc. of the 36th ICRC (Madison), 2019

[31] O. Adriani et al., ApJ 863, 160, 2018

[32] M. Mori, Y. Asaoka et al. (CALET Collaboration), Proc. of the 36th ICRC (Madison), 2019

[33] P. S. Marrocchesi et al., Nucl. Instr. Meth. A 659, 477-483, 2011

[34] Y. Asaoka et al., Astropart. Phys. 91, 1, 2017

[35] Y.Asaoka, S.Ozawa, S.Torii et al., Astropart. Phys. 100 29-37, 2018

[36] P. Maestro and N. Mori, Proc. of the 35th ICRC (Busan, Korea), 2017

[37] P. Brogi et al., Proc. of the 34th ICRC (The Hague, Netherlands), 595, 2015

[38] V. I. Zatsepin and N. V. Sokolskaya, Astronomy Astrophysics 458, 1-5, 2006

[39] A. A. Lagutin et al, J.Phys. Conf. Ser. 1181, 012023, 2019

[40] Akaike, Y.et al. (for the CALET Collaboration) 2017, Proceedings of Science (ICRC2017) 181 (8pp)

[41] Akaike, Y.et al. (for the CALET Collaboration) 2018, Proceedings of COSPAR (2018) / (6pp)

[42] O. Adriani et al. (CALET Collab.), Astrophys. J. Lett. 863, 160 (2018)

[43] O. Adriani et al. (CALET Collab.), Astrophys. J. Lett. 829, L20 (2016)

[44] N. Cannady et al. (CALET Collab.), Astrophys. J. Suppl. S. 238, 5 (2018) 